\title{
Editorial
}

\section{Living with a univentricular heart}

The Paris group of Hôpital Necker-Enfants Malades have reviewed 89 patients who have grown up with a univentricular heart, and their findings are presented in this issue of Heart. ${ }^{1}$ First we should congratulate Saliba and his colleagues for undertaking this study and providing the data. In an editorial in Heart in 1993 Fallowfield wrote " . . .cardiologists have been slow to routinely employ appropriate measures to assess quality of life."2 Since then there have been 145 papers in the British Heart fournal or in Heart with "quality of life" in the title or abstract. Cardiologists have risen to the challenge.

At the time of Saliba's survey the 89 patients ranged in age from 17-49 years with a median of 21 years, and we can deduce that the ages are bunched around the late teens and early 20s. Most have undergone palliative procedures including venous (cavopulmonary or right atriopulmonary shunts) or arterial (aortopulmonary shunt) diversion. Four have had a heart transplant and another four were on the waiting list. They were very unwell from any reasonable cardiological standpoint. We see that $10 \%$ had fixed pulmonary hypertension, $20 \%$ were in congestive heart failure, $40 \%$ had a cardiac rhythm disturbance, and $60 \%$ were cyanotic at rest.

It is then remarkable to read that "the [QoL] scores of adults with univentricular heart were similar to the normal population". Again I believe congratulations are due to the group. Not withstanding their very heavy burden of disability, these patients value their lives highly, and lead life to the full. To achieve this is good doctoring, whatever it takes in the form of surgical palliation, medical management, and morale boosting, and it should be recognised and applauded. Nevertheless we have some work to do to square the discrepancy between some robust measures of cardiac status that would predict substantial disability and what the patients perceive and report as their near normal health status.

\section{Duke questionnaire}

Those not familiar with quality of life (QoL) instruments should look at the 17 item Duke questionnaire reproduced as table 2 in Saliba's paper. ${ }^{1}$ Think of an individual from your own experience with life long disability but who is psychologically, emotionally, and socially well adjusted. For example, I have a patient now aged 65 who has been paraplegic for 40 years since an accident on an oil rig. $\mathrm{He}$ is unable to walk up a flight of stairs (question 8) and knowing him he might well label the question "nonapplicable". He might cross out "running" the length of a football pitch (question 9) because his mode of covering the ground for most of his life has been on his wheels. His high overall high score on the Duke questionnaire is not an error or wrong in any way. He does indeed have a very high QoL and has no hesitation in attesting to it. So should I then stop worrying about spinal cord protection when I operate on the descending aorta? No. Paraplegia is still a tragedy. All we have shown is the indomitable nature of the human spirit in most instances and the ability to rise above chronic disability. Saliba and colleagues put it well in their discussion where they write: "These patients probably develop coping mechanisms, easing the psychological stress and thus improving the majorities of scores in the Duke profile. It may be speculated that these patients orientate themselves towards a different set of values in every day life. Disabilities may be accepted and personal expectations recalibrated."

Conversely acute illness creates a very different response. Those in the throws of sea sickness see death as a better option than completing the voyage, and yet it is a benign self limiting malaise. I doubt if any take those pleas for death literally and the sufferer in the long term can laugh about it and bears no grudge against those who kept him alive.

These effects (response shifts, scale recalibration, and changes in values) have been studied in detail in the context of breast cancer. ${ }^{3}$ They reduce the size of quite acute changes such as well being before and during radiotherapy. In states of chronic ill health not only will people make the best of what they have but they will face high risks to stay alive, they will clutch at straws, and they will be grateful for our efforts even if they are only partially successful, completely futile, or frankly misconceived.

In 1968 when the first round of largely disappointing heart transplants was coming to a close the brilliant Peter Medawar wrote: "The transplantation of organs will be assimilated into ordinary clinical practice ... and there is no need to be philosophical about this. This will come about for the single and sufficient reason that people are so constituted that they would rather be alive than dead". ${ }^{4}$

\section{Value of QoL instruments}

The QoL instruments are probably most valuable when there is a treatment which has as many downsides as advantages, where the health gain is not immediately obvious, and there are two groups to compare. Long term treatment of hypertension is probably a reasonable example. The health gain is reduction in heart failure and stroke in years to come. The price is pill taking with side effects which might include a very wide range of manifestations including lack of energy, worsening asthma, cough, nightmares, impotence, urinary frequency, postural hypotension and others depending on which drugs are on trial.

Formally measuring QoL was also seen as essential in comparing surgery versus angioplasty for angina. Surgery may be a more certain form of relief but at a higher risk of stroke and wound complications. On the other hand the angioplasty group has more recurrence and more hospital admissions. In the event most of the QoL benefits were predicted by the exercise test. ${ }^{5}$ Those who were relieved of angina had less pain, better mobility, and reported better QoL.

So what is the place of QoL measurements to describe single groups of complex patients? We have noted elsewhere the strange effect of comparing the results obtained when the 
same QoL measures are applied to transplant and eating disorder patients. ${ }^{6}$ The nature of their health problems and needs are so different that no useful comparison can be drawn. Perhaps the same might apply in these patients coping with chronic cardiac limitation caused by adult congenital heart disease. We treat each case on its merits on the basis of the best available strategies we know.

If we reach that conclusion, why do we want QoL measurements in such cases anyway? Will they permit health economists to calculate cost efficiency? If so these patients' perceptions will seriously mislead the economists. Is it to allow planners to prioritise? For similar reasons it seems dangerous to suggest that one individual's life is more or less valuable than another's. We should applaud these excellent subjective outcomes as an indication of good holistic care in the management of these very difficult patients, but it will still be the objective measures of cardiac function that will guide us. Cardiac surgery and interventional cardiology are fundamentally mechanistic. We alter structure in order to improve function and will want more concrete evidence than the coping patient putting a brave face on it.
Cardiothoracic Unit,

TOM TREASURE

Guy's and St Thomas' Hospital,

St Thomas Street

London SE1 9RT

UK

tom.treasure@ukgateway.net

I am grateful to Professor Leslie Fallowfield for advice on the preparation of this editorial.

1 Saliba Z, Butera G, Bonnet D, et al. Quality of life and perceived health status in surviving adults with univentricular heart. Heart 2001; 86:69-73.

2 Fallowfield L. Quality of life assessment in patients with heart disease. $\mathrm{Br}$ Heart f 1993;69:371-2.

3 Jansen SJT, Stiggelbout AM, Nooij M, et al. Response shift in quality of life measurement in early-stage breast cancer patients undergoing radiotherapy. Quality of Life Research 2000;9:603-15. 2000.

4 Kantrowitz A, Haller JD. Symposium on human heart transplantation. Introduction. Am f Cardiol 1968;22:761-5.

5 Pocock SJ, Henderson RA, Seed P, et al. Quality of life, employment status, and anginal symptoms after coronary angioplasty or bypass surgery. 3-year and anginal symptoms after coronary angioplasty or bypass surgery. 3-year follow-up in the randomized

6 Keilen M, Treasure T, Schmidt U, et al. Quality of life measurements in eating disorders, angina, and transplant candidates: are they comparable? $f R$ Soc Med 1994;87:441-4.

\section{IMAGES IN CARDIOLOGY}

\section{Non-sustained ventricular tachycardia during carotid massage}

Carotid sinus massage is a useful bedside test in the evaluation of patients with syncope and in the diagnosis and possible termination of certain types of supraventricular tachycardia. The most frequent complication related to carotid massage is cerebrovascular accident, particularly in elder patients. We present a case of non-sustained ventricular tachycardia (nsVT) provoked by carotid massage.

A 41 year old female patient with a history of myocardial infarction and hypertension was referred for evaluation of repeated episodes of non-documented presyncope and syncope.

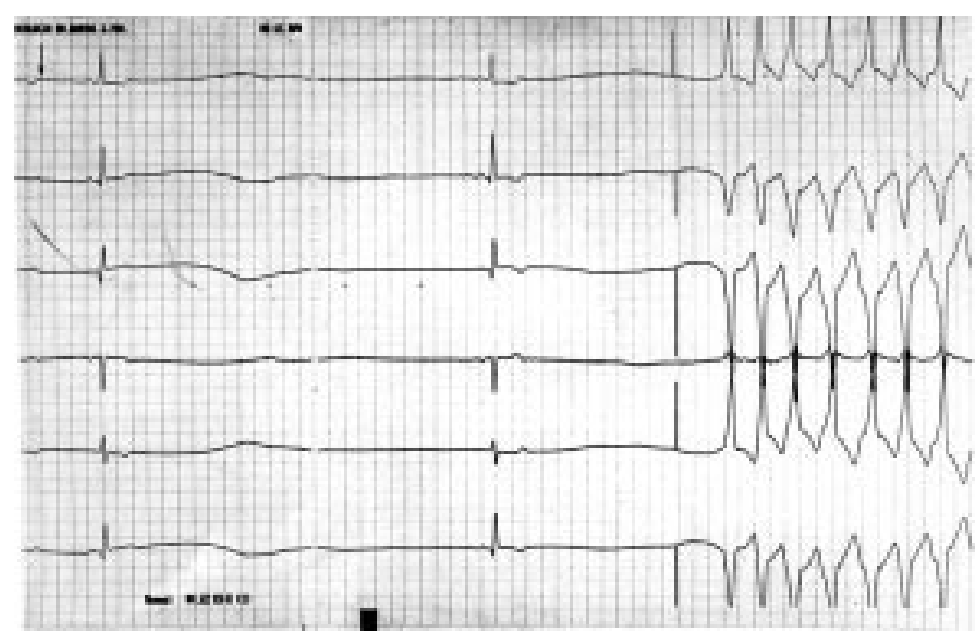

Standard 12 lead ECG showed sinus rhythm, PQ 160 ms, no bundle branch block morphology, and pathologic $Q$ waves in inferior leads. Left ventricular ejection fraction estimated by echocardiography was $40 \%$. Carotid massage resulted in a $5160 \mathrm{~ms}$ sinus pause, and the next sinus beat was followed by a monomorphic nsVT after a $3040 \mathrm{~ms}$ pause. The ventricular tachycardia lasted 9 beats at a rate of $140 \mathrm{bpm}$, and terminated spontaneously (see fig). During a subsequent 24 hour Holter monitoring an episode of asymptomatic monomorphic nsVT (11 beats, 130 beats/min) was recorded. The patient gave no consent for coronary angiography but agreed to undergo electrophysiology testing, by which only a 4 beat run of nsVT could be induced using an aggressive stimulation protocol (two stimulation sites, three drive train, up to three extrastimuli, isoproterenol). Since the syncope episodes were more consistent with bradycardia caused by carotid sinus hyperaesthesia and there was no inducible ventricular tachycardia, the patient was treated with a dual chamber pacemaker and a $\beta$ blocker.

The present case indicates that carotid massage may result in ventricular arrhythmia. If it is anticipated and prompt treatment is available, carotid massage should remain a simple and useful test.

ADAM BÖHM ARNOLD PINTÉR ISTVÁN PRÉDA 